\title{
Breaking of phase rigidity by a time-varying field for a two-terminal modified Aharonov-Bohm ring
}

\author{
Qing-feng Sun \\ Department of Physics, The University of Hong Kong, Pokfulam Road, Hong Kong, China \\ and Department of Physics, Peking University, Beijing 100871, China \\ Jian Wang \\ Department of Physics, The University of Hong Kong, Pokfulam Road, Hong Kong, China \\ Tsung-han Lin \\ Department of Physics, The University of Hong Kong, Pokfulam Road, Hong Kong, China \\ and Department of Physics, Peking University, Beijing 100871, China \\ (Received 17 May 1999; revised manuscript received 7 September 1999)
}

\begin{abstract}
For a two-terminal modified Aharonov-Bohm (AB) ring with a quantum dot inserted in one arm and threaded by a magnetic flux, the phase rigidity is observed experimentally in the linear response regime, and also established theoretically. We show that this phase rigidity can be broken, even in the linear response regime, by applying a time-varying field on the dot, together with the magnetic flux through the $\mathrm{AB}$ ring. This provides another way of observing the continuous variation of the transmission phase through a two-terminal mesoscopic system. [S0163-1829(99)50444-X]
\end{abstract}

\section{INTRODUCTION}

Recently, in a set of breakthrough experiments, Yacoby et $a{ }^{1}{ }^{1}$ and Schuster et $a l .{ }^{2}$ successfully performed the measurements of the transmission phase through a mesoscopic modified Aharonov-Bohm (AB) ring, with a quantum dot (QD) inserted in one arm and a magnetic flux threaded through the center of the ring. In addition to the flux as a source of phase shift in the bare AB ring, the inserted QD, operating in the Coulomb blockade $(\mathrm{CB})$ regime, becomes another source of phase shift. In tunneling through the QD, an electron acquires an additional phase, leading to a shift in the $\mathrm{AB}$ oscillations. ${ }^{3}$ By varying the gate voltage $\mathrm{v}_{g}$, one can measure this phase shift as a function of $\mathrm{v}_{g}$. Surprisingly, instead of a continuous variation of this phase shift, in the experiment of Yacoby et al., ${ }^{1}$ they found a bimodal behavior: the phase shift is zero for all values of $\mathrm{v}_{g}$ on one side of the CB resonance and $\pi$ for all values on the other side. ${ }^{3}$ This peculiar behavior, known as the phase rigidity, has been studied extensively. ${ }^{4-7}$

This phase rigidity is a general property coming from the two-terminal nature of the setup in the measurement by Yacoby et al., which is based on time-reversal symmetry and current conservation. ${ }^{8}$ In fact, time-reversal symmetry requires $t_{L R}(\epsilon, \phi)=t_{R L}(\epsilon,-\phi)$, where $t_{\alpha \beta}(\epsilon, \phi)(\alpha, \beta=L, R)$ is the transmission amplitude from terminal $\alpha$ to $\beta, \epsilon$ is the energy of the electron, and $\phi=2 \pi \Phi / \Phi_{0}\left(\Phi_{0}=h / e\right)$. From current conservation (unitary condition of scattering matrix ), one has $\left|t_{L R}(\epsilon, \phi)\right|^{2}=\left|t_{R L}(\epsilon, \phi)\right|^{2} .8$ Then one finds $\left|t_{L R}(\epsilon, \phi)\right|^{2}=\left|t_{L R}(\epsilon,-\phi)\right|^{2}$. Namely, the transmission probability $\left|t_{L R}(\epsilon, \phi)\right|^{2}$ is an even function of $\phi$. Consequently, the linear conductance $G$ (and the current) is also an even function of $\phi$, and its Fourier expansion is

$$
G(\phi)=G_{0}+\sum_{n} G_{n} \cos \left(n \phi+\delta \phi_{n}\right)
$$

Obviously, the phase shift $\delta \phi_{n}(n=1,2, \ldots)$ can only take two values, either 0 or $\pi$. Namely, the phase of the twoterminal $\mathrm{AB}$ ring has to be rigid, or change abruptly by $\pi$ as the accumulated phase in one arm is being varied. ${ }^{6}$

Is it possible to obtain a continuous phase variation for a two-terminal $\mathrm{AB}$ ring? It cannot be a simple $\mathrm{AB}$ ring of course, but modified to include an inelastic scattering channel so that the unitary condition for the scattering matrix, one of the conditions mentioned above, will not lead to phase rigidity. With this idea in mind, we consider in this paper a two-terminal modified $\mathrm{AB}$ ring threaded by a magnetic flux and with a QD inserted in one arm. To introduce the inelastic scattering, we apply a time-varying microwave (MW) field on the QD. In the presence of MW field, the time averaged current is given by

$$
\begin{aligned}
\bar{I}= & e^{2} / h \int d \epsilon \sum_{n}\left[\left|t_{L R}(\epsilon, \epsilon+n \hbar \omega, \phi)\right|^{2} f_{L}\right. \\
& \left.-\left|t_{R L}(\epsilon, \epsilon+n \hbar \omega, \phi)\right|^{2} f_{R}\right],
\end{aligned}
$$

where $\omega$ is the frequency of the MW field, $t_{L R}(\epsilon, \epsilon$ $+n \hbar \omega, \phi)$ is the transmission amplitude for electrons coming from the left lead with energy $\epsilon$, and to the right with energy $\epsilon+n \hbar \omega$. The time-reversal symmetry ensures $t_{R L}(\epsilon, \epsilon+n \hbar \omega, \phi)=t_{L R}(\epsilon+n \hbar \omega, \epsilon,-\phi)$. However, to maintain the phase rigidity one must require $\Sigma_{n} \mid t_{R L}(\epsilon, \epsilon$ $+n \hbar \omega, \phi)\left.\right|^{2}=\Sigma_{n}\left|t_{L R}(\epsilon+n \hbar \omega, \epsilon, \phi)\right|^{2}$, which is not valid in the presence of an inelastic channel. To see this, we examine the following equations from the unitary condition of a scattering matrix: $\quad \Sigma_{n}\left|r_{L L}(\epsilon, \epsilon+n \hbar \omega, \phi)\right|^{2}+\Sigma_{n} \mid t_{L R}(\epsilon, \epsilon$ $+n \hbar \omega, \phi)\left.\right|^{2}=1 \quad$ and $\quad \Sigma_{n}\left|r_{L L}(\epsilon+n \hbar \omega, \epsilon, \phi)\right|^{2}+\Sigma_{n} \mid t_{R L}(\epsilon$ $+n \hbar \omega, \epsilon, \phi)\left.\right|^{2}=1$, where $r$ is the reflection amplitude. In the absence of the MW field, these two equations give $\left|t_{R L}(\epsilon, \phi)\right|^{2}=\left|t_{L R}(\epsilon, \phi)\right|^{2}$ which is needed for the phase ri- 
gidity. When the MW field is present, similar relations do not hold any more. Therefore, the phase rigidity is broken, even for a two-terminal modified $\mathrm{AB}$ ring. By using the nonequilibrium Green's function (NGF) method, we obtained the time-averaged current $\bar{I}$ and the linear differential conductance $d \bar{I} / d V$, and found that $d \bar{I} / d V$ as a function of $\phi$ is a periodic but not an even function. Consequently, for this two-terminal modified $\mathrm{AB}$ ring, the phase shift of the oscillation, $\delta \phi_{1}$, can vary continuously.

It is worth mentioning that a continuous phase variation observed in the linear response regime in the experiment by Schuster et al. ${ }^{2}$ is due to the four-terminal nature of the setup, which relaxes the symmetry requirements, so that all values of phase shift are allowed. ${ }^{3}$ Also note that in the theory of Bruder, Fazio, and Schoeller, ${ }^{7}$ they obtained a continuous phase variation for a two-terminal modified $\mathrm{AB}$ ring, in which the system is in nonlinear response regime (i.e., finite bias voltage) and the QD is strongly correlated. In contrast, the above mentioned phase rigidity is related only to the linear response regime.

\section{MODEL AND FORMULATION}

The system under consideration is a two-terminal modified $\mathrm{AB}$ ring threaded by a magnetic flux $\Phi$ and with a quantum dot inserted in one arm. To simplify the calculation but still capture the essential physics, we shall restrict ourselves to considering the tunneling in the neighborhood of a single Coulomb oscillation peak. Therefore, we assume that only one nondegenerate electronic energy level in the QD is involved, and the spin degree of freedom of the electron and the intradot Coulomb interaction can be neglected, as in Ref. 9. The Hamiltonian of the system is written as

$$
\begin{aligned}
H(t)= & \sum_{k \in L} \epsilon_{k} a_{k}^{\dagger} a_{k}+\sum_{p \in R} \epsilon_{p} b_{p}^{\dagger} b_{p}+\epsilon_{0} c_{0}^{\dagger} c_{0}+\epsilon_{1}(t) c_{1}^{\dagger} c_{1} \\
& +\left\{\sum_{k, j} w_{k}^{j} a_{k}^{\dagger} c_{j}+\sum_{p} w_{p}^{0} b_{p}^{\dagger} c_{0}+\sum_{p} w_{p}^{1} e^{i \phi} b_{p}^{\dagger} c_{1}\right. \\
& + \text { H.C. }\},
\end{aligned}
$$

where $a_{k}^{\dagger}\left(a_{k}\right)$ and $b_{p}^{\dagger}\left(b_{p}\right)$ are the creation (annihilation) operators of the electron in the left and right lead, respectively. The third term describes the bare arm of the ring. The fourth term models the quantum dot which is regulated by a gate voltage $\mathrm{v}_{g}$. Here we assume that a time-varying MW field is applied on the quantum dot. We also assume that the adiabatic approximation holds so that the MW field can be described by an oscillating potential and it only causes the single-electron energy spectrum a rigid shift: ${ }^{10,11} \epsilon_{1}(t)=\epsilon_{1}$ $+\Delta \cos \omega t, \epsilon_{1}$ is the time-independent single electron energy without the MW field. The last term in Eq. (2) represents the tunneling between the ring and the leads. To account for the magnetic flux $\Phi$ through the center of the $\mathrm{AB}$ ring, the matrix elements between the dot and the right lead is set to be $w_{p}^{1} e^{i \phi}$ as in Ref. 5, where $w_{p}^{1}$ is the matrix element in the absence of the magnetic field, and $\phi=2 \pi \Phi / \Phi_{0}$.

It should be mentioned that Jauho and Wingreen investigated, very recently, the phase shift of the transmission am- plitude for an electron through a quantum dot applied by a MW field, ${ }^{9}$ but their main interest is focused on a simple two-terminal system (a dot coupled to two leads), which is not relevant to the problem of phase rigidity.

The current from the left lead into the system can be calculated from the evolution of the total number operator of the electrons of the left lead. Then one finds (in units of $\hbar$ $=1)^{10,11}$

$$
\begin{aligned}
I_{L}(t)= & -2 e \operatorname{Im} \int_{-\infty}^{t} d t_{1} \int \frac{d \boldsymbol{\epsilon}}{2 \pi} e^{-i \epsilon\left(t_{1}-t\right)} \operatorname{Tr}\left\{\boldsymbol{\Gamma}^{L}(\boldsymbol{\epsilon})\right. \\
& \left.\times\left[\mathbf{G}^{<}\left(t, t_{1}\right)+f_{L}(\boldsymbol{\epsilon}) \mathbf{G}^{r}\left(t, t_{1}\right)\right]\right\},
\end{aligned}
$$

in which $f_{L(R)}(\epsilon)=\left\{\exp \left[\left(\epsilon-e V_{L(R)}\right) / k_{B} \mathcal{T}\right]+1\right\}^{-1}$ is the Fermi distribution function of electrons in the left (right) lead, and $\Gamma^{L}(\epsilon)$ is a matrix linewidth function defined by $\Gamma_{i j}^{L(R)}$ $\equiv \Sigma_{k(p)} 2 \pi \delta\left(\epsilon-\epsilon_{k(p)}\right) w_{k(p)}^{i *} w_{k(p)}^{j}$, where $i, j=0,1$. The matrix Green's function $\mathbf{G}^{r,<}\left(t, t_{1}\right)$ in Eq. (3) is defined by $G_{i j}^{r}\left(t, t_{1}\right)=-i \theta\left(t-t_{1}\right)\left\langle\left\{c_{i}(t), c_{j}^{\dagger}\left(t_{1}\right)\right\}\right\rangle \quad$ and $\quad G_{i j}^{<}\left(t, t_{1}\right)$ $=i\left\langle\left\{c_{j}^{\dagger}\left(t_{1}\right) c_{i}(t)\right\}\right\rangle$.

We now proceed to solve the Green's functions $\mathbf{G}^{<}\left(t, t^{\prime}\right)$ and $\mathbf{G}^{r}\left(t, t^{\prime}\right)$. First, we solve the retarded Green's functions $\mathbf{G}^{r}\left(t, t^{\prime}\right)$ by using the Dyson equation. By taking the widebandwidth approximation, which has been widely used in mesoscopic systems, ${ }^{10,11}$ the linewidth function $\Gamma^{L}\left(\Gamma^{R}\right)$ becomes independent of the energy $\epsilon$; and the self-energy $\mathbf{\Sigma}^{r}\left(t, t^{\prime}\right)$ can be obtained as follows:

$$
\boldsymbol{\Sigma}^{r}\left(t, t^{\prime}\right)=-\frac{i}{2} \delta\left(t-t^{\prime}\right)\left(\begin{array}{ll}
\Gamma_{00} & \Gamma_{01}^{L}+\Gamma_{01}^{R} e^{i \phi} \\
\Gamma_{10}^{L}+\Gamma_{10}^{R} e^{-i \phi} & \Gamma_{11}
\end{array}\right),
$$

where $\Gamma_{j j}=\Gamma_{j j}^{L}+\Gamma_{j j}^{R}$. The quantity $\mathbf{g}^{r}\left(t, t^{\prime}\right)$ in the Dyson equation describes the Green's function for the electron in the ring when the coupling between the ring and the two leads is off. It can be obtained exactly:

$$
g_{i j}^{r}\left(t, t^{\prime}\right)=-i \theta\left(t-t^{\prime}\right) \delta_{i j} \exp \left[-i \int_{t^{\prime}}^{t} d \tau \epsilon_{j}(\tau)\right],
$$

where $i, j=0,1$. It should be emphasized that the Green's function $\mathbf{g}^{r}\left(t, t^{\prime}\right)$ depends on two time variables $t$ and $t^{\prime}$, not their difference. In order to solve the Dyson equation, we take the Fourier transform ${ }^{12}$

$$
F\left(t, t_{1}\right)=\sum_{n} e^{i n \omega t_{1}} \int \frac{d \epsilon}{2 \pi} e^{-i \epsilon\left(t-t_{1}\right)} F_{n}(\epsilon),
$$

where $F$ represents any one of the $\mathbf{G}^{r}, \mathbf{g}^{r}$, or $\boldsymbol{\Sigma}^{r}$, and $\omega$ is the frequency of the MW field. Denoting $F_{m n}(\epsilon) \equiv F_{n-m}(\epsilon$ $+m \omega)$, the Fourier transformation of the Green's function $\mathbf{g}^{r}\left(t, t^{\prime}\right)$ and the self-energy $\boldsymbol{\Sigma}^{r}\left(t, t^{\prime}\right)$ are then easily obtained, and the Dyson equation becomes $\mathbf{G}_{m n}^{r}=\mathbf{g}_{m n}^{r}$ $+\sum_{k} \mathbf{g}_{m k}^{r} \boldsymbol{\Sigma}_{k k}^{r} \mathbf{G}_{k n}^{r}$, in which the argument $\boldsymbol{\epsilon}$ has been suppressed. By iterating the Dyson equation, and taking the approximation,

$$
\sum_{n, m}\left(E_{n}^{0} E_{m}^{0}\right)^{-1} \approx \sum_{n, m} \delta_{n m} /\left(E_{m}^{0}\right)^{2}
$$


where $E_{l}^{j} \equiv \epsilon-\epsilon_{j}+l \omega+i \Gamma_{j j} / 2(j=0,1)$, which is reasonable for $\omega \gg \Gamma_{00}$. We then obtain matrix elements of the Green's function $\mathbf{G}_{m n}^{r}(\boldsymbol{\epsilon})$ as

$$
\begin{gathered}
G_{m n ; 00}^{r}=\frac{\delta_{m n}}{\left(\widetilde{g}_{m m ; 00}^{r}\right)^{-1}-\sum_{m m ; 01}^{r} \widetilde{g}_{m m ; 11}^{r} \Sigma_{m m ; 10}^{r}}, \\
G_{m n ; 01}^{r}=G_{m m ; 00}^{r} \Sigma_{m m ; 01}^{r} \widetilde{g}_{m n ; 11}^{r}, \\
G_{m n ; 10}^{r}=\widetilde{g}_{m n ; 11}^{r} \Sigma_{n n ; 10}^{r} G_{n n ; 00}^{r}, \\
G_{m n ; 11}^{r}=\widetilde{g}_{m n ; 11}^{r}+\sum_{k} \widetilde{g}_{m k ; 11}^{r} \Sigma_{k k ; 10}^{r} G_{k k ; 00}^{r} \sum_{k k ; 01}^{r} \widetilde{g}_{k n ; 11}^{r},
\end{gathered}
$$

in which $\quad \tilde{g}_{m n ; 00}^{r}(\epsilon)=\delta_{m n} / E_{m}^{0} \quad$ and $\quad \tilde{g}_{m n ; 11}^{r}(\epsilon)$ $=\Sigma_{k} J_{k+m}(\Delta / \omega) J_{k+n}(\Delta / \omega) / E_{-k}^{1}$. By taking the reverse Fourier transformation of Eq. (7), the Green's function $\mathbf{G}^{r}\left(t, t^{\prime}\right)$ can be obtained immediately.

The next step is to solve the distribution Green's function $\mathbf{G}^{<}(t, t)$ by using the Keldysh equation: $\mathbf{G}^{<}(t, t)$ $=\iint d t_{1} d t_{2} \mathbf{G}^{r}\left(t, t_{1}\right) \mathbf{\Sigma}<\left(t_{1}, t_{2}\right) \mathbf{G}^{a}\left(t_{2}, t\right)$. Under the widebandwidth approximation, the self-energy $\mathbf{\Sigma}<\left(t_{1}, t_{2}\right)$ becomes

$$
\boldsymbol{\Sigma}^{<}\left(t_{1}, t_{2}\right)=\int \frac{d \boldsymbol{\epsilon}}{2 \pi} i e^{-i \epsilon\left(t_{1}-t_{2}\right)}\left[f_{L}(\boldsymbol{\epsilon}) \boldsymbol{\Gamma}^{L}+f_{R}(\boldsymbol{\epsilon}) \widetilde{\mathbf{I}}^{R}(\phi)\right],
$$

where

$$
\widetilde{\mathbf{I}}^{R}(\phi)=\left(\begin{array}{ll}
\Gamma_{00}^{R} & \Gamma_{01}^{R} e^{i \phi} \\
\Gamma_{10}^{R} e^{-i \phi} & \Gamma_{11}^{R}
\end{array}\right) .
$$

Substituting $\mathbf{G}^{r}\left(t, t_{1}\right)$ and $\mathbf{\Sigma}<\left(t_{1}, t_{2}\right)$ into the Keldysh equation, one easily obtains $\mathbf{G}^{<}\left(t, t^{\prime}\right)$. Finally, substituting the Green's functions $\mathbf{G}^{<}(t, t)$ and $\mathbf{G}^{r}\left(t, t^{\prime}\right)$ into Eq. (3), the time-dependent current $I_{L}(t)$ can be obtained straightforwardly. Hence, the time-averaged current $\bar{I}$ is obtained as follows:

$$
\begin{aligned}
\bar{I}= & -e \int \frac{d \boldsymbol{\epsilon}}{2 \pi} \operatorname{Tr}\left\{2 \operatorname{Im}\left[\boldsymbol{\Gamma}^{L} \mathbf{G}_{00}^{r}(\boldsymbol{\epsilon}) f_{L}(\boldsymbol{\epsilon})\right]\right. \\
& +\operatorname{Re} \sum_{n} \boldsymbol{\Gamma}^{L} \mathbf{G}_{-n 0}^{r}(\boldsymbol{\epsilon})\left[f_{L}(\boldsymbol{\epsilon}) \boldsymbol{\Gamma}^{L}+f_{R}(\boldsymbol{\epsilon}) \widetilde{\boldsymbol{\Gamma}}^{R}(\phi)\right] \\
& \left.\times\left[\mathbf{G}_{-n 0}^{r}(\boldsymbol{\epsilon})\right]^{\dagger}\right\} .
\end{aligned}
$$

The expression of the averaged current $\bar{I}$, Eq. (9), is the central result of this work. It can be applied to the arbitrary strength of the time-dependent MW field. Note that, by the way, if one decouples the connection to the bare arm of the ring (i.e., let $w_{k}^{0}=w_{p}^{0}=0$ ), then the system will reduce to a simple two-terminal system with one dot coupled to two leads, and our averaged current formula, Eq. (9), will reduce to the same result of Ref. 11 .

\section{NUMERICAL STUDIES}

Now let us apply the averaged current formula, Eq. (9), only in the linear response regime, to investigate the dependence of $\bar{I}$ on $\mathrm{\vee}_{g}\left[\mathrm{\vee}_{g}\right.$ regulates the energy level of the quantum dot by $\left.\epsilon_{1}\left(\mathrm{~V}_{g}\right)=\epsilon_{1}(0)-e \mathrm{~V}_{g}\right]$ and $\phi$, and through that

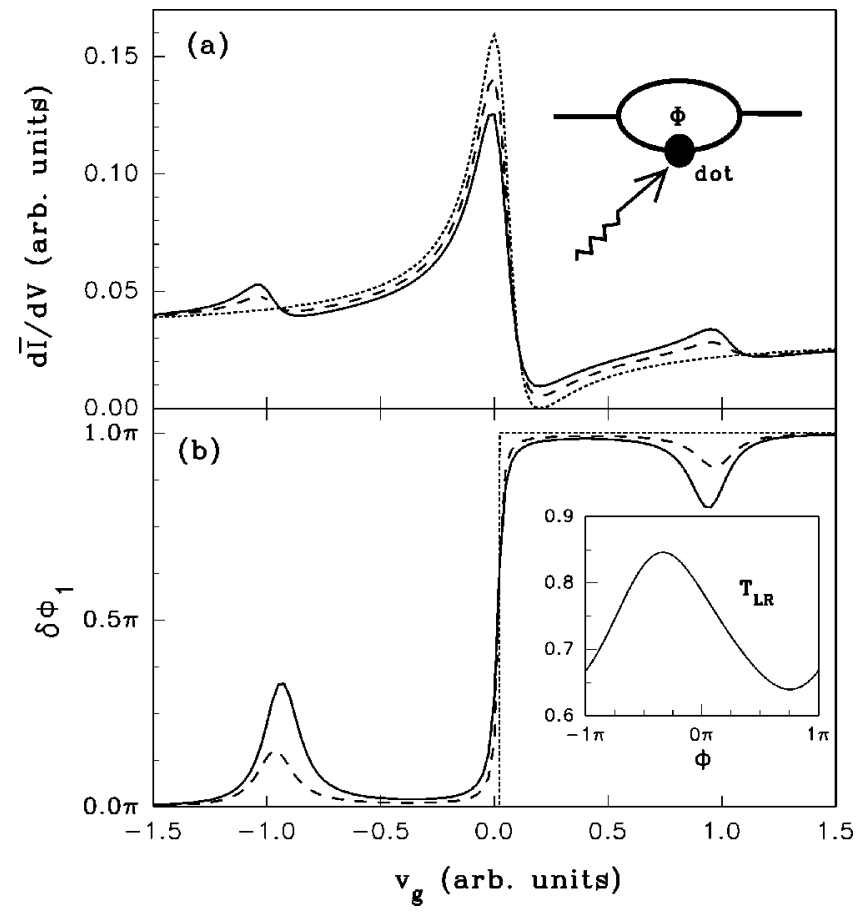

FIG. 1. (a) The linear conductance $d \bar{I} / d V$ vs the gate voltage $\mathrm{v}_{g}$ for $\phi=0$. (b) The phase $\delta \phi_{1} \mathrm{vs}_{g}$. Where $\omega=1, \Gamma_{00}^{L}=\Gamma_{00}^{R}=\Gamma_{11}^{L^{g}}$ $=\Gamma_{11}^{R}=0.1, \epsilon_{0}=0.2$, and $\epsilon_{1}=0$ at $\mathrm{v}_{g}=0$. The solid, dashed, and dotted curves correspond to $\Delta / \omega=0.7,0.5$, and 0 , respectively. The inset in (a) is a schematic diagram of the two-terminal modified AB ring. The inset in (b) is the transmission probability $T_{L R}$ vs $\phi$ at zero gate voltage $\left(\mathrm{v}_{g}=0\right)$ and $\epsilon=0$. The other parameters are the same as the solid curve.

obtain the behavior of the phase shift $\delta \phi_{1}$. In numerical studies, we take the units of $e=\hbar=1$, and make further simplifications as follows: (i) Consider two symmetric barriers, i.e., $\Gamma_{00}^{L}=\Gamma_{00}^{R}$ and $\Gamma_{11}^{L}=\Gamma_{11}^{R}$; (ii) temperature $\mathcal{T}=0$; (iii) set $V_{R}=0$ for the right lead, which does not change the physics due to the gauge invariance. ${ }^{13}$ It should be emphasized that the nondiagonal linewidths $\Gamma_{01}^{L(R)}$ and $\Gamma_{10}^{L(R)}$ are associated with the diagonal linewidths through the relation $\Gamma_{01}^{L(R)} \Gamma_{10}^{L(R)}=\Gamma_{00}^{L(R)} \Gamma_{11}^{L(R)}$. Therefore, in numerical calculation, one only needs to choose the diagonal linewidth parameters $\Gamma_{00}^{L(R)}$ and $\Gamma_{11}^{L(R)}$.

Figure 1(a) shows the linear conductance $d \bar{I} / d V$ vs the gate voltage $\mathrm{v}_{g}$. In the absence of the MW field (the dotted curve), a resonance peak with slight asymmetry emerges. The asymmetry structure is from the interference between $G_{i j}^{r}(i, j=0,1)$ and the Green's function of leads. This interference is a Fano-like interference as mentioned in the Kondo system by Thimm et al. ${ }^{14,15}$ In fact, without MW field and $\phi=0$, we have $d \bar{I} / d V=e / 2 \pi(x+p)^{2} /\left[p^{2}+2 p x+x^{2}(1\right.$ $\left.\left.+p^{2}\right)\right]$, with $x=\left(\epsilon_{0}-\epsilon\right) /\left(\Gamma_{00} / 2\right)$ and $p=\left(\epsilon_{1}-\epsilon\right) /\left(\Gamma_{11} / 2\right)$. While in the presence of the MW field (the solid and dashed curves), some additional peaks show up at the locations of $n \hbar \omega(n= \pm 1, \pm 2, \ldots)$ away from the original resonance peak, indicating the photon-assisted tunneling (PAT) processes. The additional peaks are also slightly asymmetric due to the sideband of level $\epsilon_{0}$ participating in the Fano-like interference also.

The behavior of the phase shift of the linear conductance 


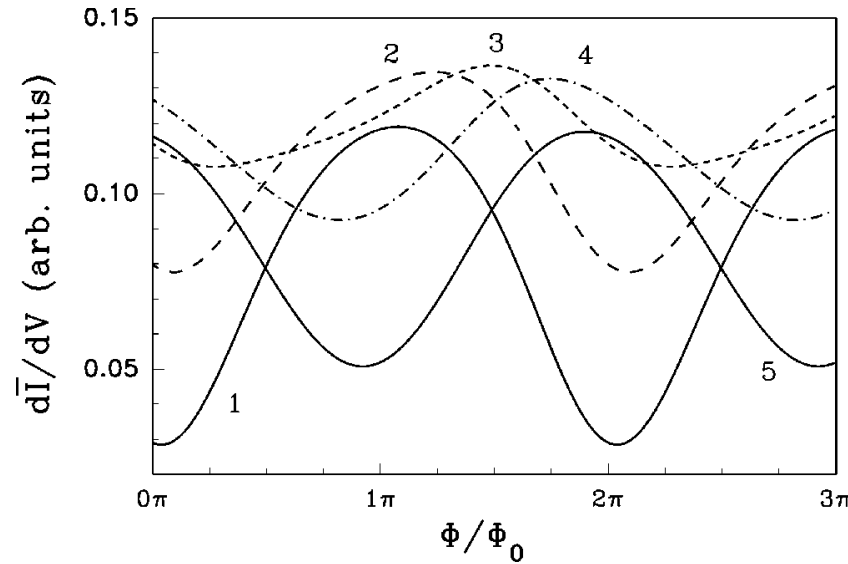

FIG. 2. The conductance $d \bar{I} / d V$ vs $\phi$ at these $\mathrm{v}_{g}$ around the original resonance peak in the solid curve at Fig. 1(a). The parameters are same with the solid curve at Fig. 1(a). Curve 1 to curve 5 corresponds to $\mathrm{v}_{g}=0.1,0.05,0.02,-0.01$, and -0.05 , respectively.

$d \bar{I} / d V$ vs $\phi$ is presented in Fig. 2, showing the conductance $d \bar{I} / d V$ vs $\phi$ for different gate voltage $\mathrm{v}_{g}$, around the original resonance peak corresponding to different points in the solid curve of Fig. 1(a). All curves exhibit periodic oscillations with the period of $2 \pi$, but with different phase shifts. One can clearly see that the first-order phase shift $\delta \phi_{1}$ of the oscillations does have a continuous variation, not taking only two values (either $o$ or $\pi$ ), i.e., the phase rigidity is broken. By taking a Fourier expansion for $d \bar{I} / d V$ vs $\phi$ as in Eq. (1), the phase shift $\delta \phi_{1}$ can be obtained analytically, which is plotted in Fig. 1(b). Without the MW field (the dotted curve), the phase shift is rigid and an abrupt phase increases, by $\pi$, on passing through the resonance peak. These results are in complete agreement with the experiment ${ }^{1}$ and the previous theoretical results. $^{4-7}$ In the presence of the MW field (the solid and dashed curves), however, the phase can vary continuously, i.e., the phase rigidity is broken. From the numerical results one can clearly see the following features for this continuous phase variation: (i) The phase shift $\delta \phi_{1}$ continuously rises by almost $\pi$ for $\mathrm{v}_{g}$ across a resonance peak. The rate of the rising decreases with the increase of $\Delta / \omega$. (ii) At $\mathrm{v}_{g}=\epsilon_{1} \mp \hbar \omega$, there is a peak (or a dip), corresponding to a photon absorption (or emission), with a half-peak-width determined by the linewidth $\Gamma_{11}$.

Physically, the phase rigidity breaking for our twoterminal modified $\mathrm{AB}$ ring in a linear response regime originates from the inelastic scattering which introduces a degree of freedom in the scattering process. In the presence of the MW field, the electron tunneling through the quantum dot can exchange the energy of $\pm n \hbar \omega(n=1,2, \ldots)$ with the MW fields, leading to the opening of inelastic tunneling channels. An incident electron from the left lead with the energy $\epsilon$ tunneling into the QD, can scatter (transmit or reflect) into an outgoing channel with the energy of $\epsilon^{\prime}=\epsilon$ $\pm n \hbar \omega\left(\epsilon^{\prime} \neq \epsilon\right)$, by absorbing or emitting $n$ photons. Consequently, the transmission probability $T_{L R} \equiv \Sigma_{n} \mid t_{L R}(\epsilon, \epsilon$ $+n \hbar \omega, \phi)\left.\right|^{2}$ will not be an even function of $\phi$. This is clearly shown in the inset of Fig. 1(b), where we have plotted transmission probability $T_{L R}$ vs $\phi$ for a specific energy $\epsilon$ $=0$. Correspondingly, the differential conductance $d \bar{I} / d V$ is no longer an even function of $\phi$, and hence the phase shift $\delta \phi_{1}$ can vary continuously.

\section{SUMMARY}

We have investigated the phase shift behaviors in a twoterminal modified $\mathrm{AB}$ ring with a quantum dot inserted in one arm. We showed that the phase rigidity can be broken by applying a MW field on the dot, and a continuous phase shift can be obtained through the measurement of the linear conductance for this two-terminal AB system. These behaviors are attributed to the inelastic scattering channel introduced by the MW field applied to the dot.

\section{ACKNOWLEDGMENTS}

We gratefully acknowledge the financial support of a CRCG grant from the University of Hong Kong, the research grant from the Chinese National Natural Science Foundation, and the Institution of Higher Education.
${ }^{1}$ A. Yacoby et al., Phys. Rev. Lett. 74, 4047 (1995).

${ }^{2}$ R. Schuster et al., Nature (London) 385, 417 (1997).

${ }^{3}$ B. G. Levi, Phys. Today 50 (1), 19 (1997).

${ }^{4}$ A. L. Yeyati and M. Büttiker, Phys. Rev. B 52, 14360 (1995).

${ }^{5}$ G. Hackenbroich and H. A. Weidenmüller, Phys. Rev. Lett. 76, 110 (1996); Phys. Rev. B 53, 16379 (1996).

${ }^{6}$ A. Yacoby, R. Schuster, and M. Heiblum, Phys. Rev. B 53, 9583 (1996).

${ }^{7}$ C. Bruder, R. Fazio, and H. Schoeller, Phys. Rev. Lett. 76, 114 (1996).

${ }^{8}$ M. Büttiker, Phys. Rev. Lett. 57, 1761 (1986).
${ }^{9}$ A. P. Jauho and N. S. Wingreen, Phys. Rev. B 58, 9619 (1998).

${ }^{10}$ N. S. Wingreen, A.-P. Jauho, and Y. Meir, Phys. Rev. B 48, 8487 (1993); A.-P. Jauho, N. S. Wingreen, and Y. Meir, ibid. 50, 5528 (1994).

${ }^{11}$ Q.-f. Sun and T.-h. Lin, Phys. Rev. B 56, 3591 (1997); J. Phys.: Condens. Matter 9, 4875 (1997).

${ }^{12}$ J. C. Cuevas, A. Martin-Rodero, and A. L. Yeyati, Phys. Rev. B 54, 7366 (1996).

${ }^{13}$ Z. S. Ma, J. Wang, and H. Guo, Phys. Rev. B 57, 9108 (1998).

${ }^{14}$ W. B. Thimm et al., Phys. Rev. Lett. 82, 2143 (1999).

${ }^{15}$ U. Fano, Phys. Rev. 124, 1866 (1961). 